\title{
Aufruf zu einer neuen Medizin der Zwischenmenschlichkeit
}

\author{
Sabine Vuilleumier-Koch \\ Dr. med., Fachärztin für Psychiatrie und Psychotherapie, FMH
}

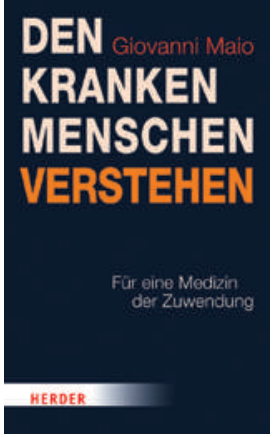

Giovanni Maio Den kranken Menschen verstehen

Für eine Medizin der Zuwendung Freiburg im Breisgau: Verlag Herder; 2015 224 Seiten, $23.90 \mathrm{CHF}$. ISBN 978-3-451-30687-7

\footnotetext{
Korrespondenz: Dr. med. Sabine Vuilleumier-Koch Fachärztin für Psychiatrie und Psychotherapie Tumigerstr. 71 CH-8606 Greifensee
}

Wer innerlich einen Schritt vom klinischen Alltag zurücktritt und sich bei der Lektüre des Buches auf die Gedanken von Giovanni Maio einlässt, gewinnt viel für die ärztliche Arbeit mit Patientinnen und Patienten. Er gewinnt das Bewusstsein für die Bedeutung der zwischenmenschlichen Begegnung zurück, das im heutigen Wald von Algorithmen und Dokumentationspflichten zu verschwinden droht. Giovanni Maio weiss als langjährig klinisch tätiger Internist und Philosoph um die Bedeutung von Vertrauen, Beziehung und Hoffnung für den kranken Menschen. Er möchte den professionellen Helfern Mut machen, diese zentralen "Werkzeuge» auch in einem "durchrationalisierten Gesundheitswesen" nicht aus der Hand zu geben.

In Geschäftsmodell Gesundheit legte Maio die verheerenden Folgen der Industrialisierung und Ökonomisierung des Gesundheitswesens auf die ärztliche Tätigkeit dar. Treten der finanzielle Aspekt des Handelns, das Ringen um mehr Umsatz, "gute» Zahlen, die Konkurrenz mit anderen "Leistungserbringern" immer mehr in den Vordergrund, so leidet die Kernaufgabe des Helfers und er hat keine Zeit mehr für den so wichtigen Dialog mit dem Patienten.

In seinem neuen Werk knüpft Maio an diese Überlegungen an, geht dann aber weiter. Es erscheint ihm wichtig, "darauf hinzuweisen, dass es die Medizin, die sich kranke Menschen erhoffen, noch immer gibt, aber sie befindet sich immer mehr in der Defensive, da sie nach Kriterien bewertet wird, die mit Zuwendung, Verstehen und Begleiten kaum noch etwas zu tun haben». Maio entwickelt die Praxis einer Medizin der Zuwendung, die den anthropologischen Grundvoraussetzungen unseres Daseins Rechnung trägt. Das Angewiesensein auf andere ist natürlich und dauert - in unterschiedlicher Ausprägung - ein ganzes Leben lang. In allgemeinverständlicher Sprache und anhand von vielen Beispielen aus der Praxis vermittelt Giovanni Maio «eine kleine Phänomenologie des Krankseins». Dabei stellt er differenzierte Betrachtungen zur Befindlichkeit von Menschen mit chronischem Schmerz, Krebs, Demenz und des sterbenden Menschen an und leitet daraus hilfreiche Wege eines Lebens mit diesen Leiden ab.

Im Umgang mit dem chronischen Schmerz sieht Maio in der modernen Medizin zwei Tendenzen, die beide den Patienten mit seinem Schmerz alleine lassen: Schmerzen als etwas "grundsätzlich Überwindbares und Vermeidbares» oder als rein kognitives Phänomen, das dem Einzelnen und seiner richtigen Einstellung überlassen wird. Machbarkeitsvorstellungen einer einseitig naturwissenschaftlich-technisch orientierten Gesellschaft haben den Mythos hervorgebracht, dass alles kontrollierbar sei, auch der Schmerz. Nach Giovanni Maio kann eine sinnvolle Therapie des Schmerzes nur darin bestehen, dem Patienten «das zurückzugeben, was ihm der Schmerz und seine soziale Umwelt immer wieder aufs Neue nehmen: das Gefühl der Wertigkeit seines Lebens, das Gefühl, Reste von Handlungsfähigkeit, Reste von Freiheiten in sich zu tragen".

Auch über den assistierten Suizid denkt Giovanni Maio in der ihm eigenen warmen, menschlichen Sprache nach. Er stellt fest, dass wir uns «stillschweigend von der Vorstellung, in einer Gemeinschaft zu leben, verabschiedet haben und glauben, dass alle Probleme des modernen Menschen, selbst die existentiellsten, allein mit seiner privaten Überzeugung zu tun haben und nichts mit der Gemeinschaft, in der er lebt». Mit der Frage, ob ein Mensch sich "wohlüberlegt» umgebracht hat, wird der Suizid zu einer "Klugheitswahl». Als Ethiker interessiert ihn die Frage der Not des einzelnen Menschen, der es überhaupt in Erwägung zieht, sich selbst zu töten, es geht ihm nicht um eine moralische Bewertung.

Giovanni Maio sieht in unserer Zeit der Durchökonomisierung des Gesundheitswesens die grosse Gefahr, dass die Medizin zunehmend das öffentliche Vertrauen verliert. Es ist schlicht unverantwortlich, die Kraft der Zuwendung stillschweigend wegzurationalisieren - das kann auf Dauer nicht gutgehen. Nehmen wir diese Worte ernst, denn es verlieren alle - Patienten und Helfer! 